\title{
53 GBd PAM-4 DAC-LESS LOW-POWER (1.5 pJ/b) SILICON INTEGRATED TRANSMITTER
}

\author{
Joris Lambrecht ${ }^{1}$, Jochem Verbist ${ }^{1,2,3}$, Hannes Ramon ${ }^{1}$, Michael Vanhoecke ${ }^{1}$, \\ Bart Moeneclaey ${ }^{1}$, Laurens Bogaert ${ }^{1,2}$, Peter Ossieur ${ }^{1}$, Joris Van Campenhout ${ }^{4}$, \\ Johan Bauwelinck ${ }^{1}$, Gunther Roelkens, ${ }^{2}$ Xin Yin ${ }^{1}$ \\ ${ }^{I}$ Ghent University-imec, IDLab, INTEC, 9052 Ghent, Belgium \\ ${ }^{2}$ Ghent University - imec, Photonics Research Group, INTEC, 9052 Ghent, Belgium \\ ${ }^{3}$ Current affiliation: BiFAST, 9000 Ghent, Belgium \\ ${ }^{4}$ imec, Silicon Photonics group, 3001 Leuven, Belgium \\ joris.lambrecht@ugent.be
}

Keywords: TRANSMITTER, PAM-4, SILICON PHOTONICS, ELECTRO-ABSORPTION MODULATOR

\begin{abstract}
We present an integrated PAM-4 transmitter consisting of two parallel GeSi electro-absorption modulators wirebonded to a SiGe BiCMOS driver, consuming 160mW excluding laser. 53GBd PAM-4 transmission is demonstrated over more than $1 \mathrm{~km}$ fiber and $2 \mathrm{~km}$ non-zero dispersion-shifted fiber, without using equalization or digital signal processing.
\end{abstract}

\section{Introduction}

Recent 400 Gigabit Ethernet (GbE) standards, e.g. 400GBASE-DR4, specify 53.125 GBd PAM-4 per lane up to $500 \mathrm{~m}$ standard single-mode fiber (SSMF) for datacenter optical interconnects [1]. Longer fiber reaches up to $10 \mathrm{~km}$ are proposed in e.g. the 400GBASE-FR4 specifications [2]. These applications require high-bandwidth, linear PAM-4 transceivers in a very compact form factor and with low power consumption. Several PAM-4 transmitters have been demonstrated at or above $50 \mathrm{GBd}$ [3-12]. Furthermore ref. [5] demonstrates a real-time 56 GBd PAM-4 link up to $2 \mathrm{~km}$ SSMF, but at $8.6 \mathrm{~W}$ power consumption (excluding laser). However, [3-5] are based on long, travelling-wave MachZehnder modulators (MZMs), which typically occupy a lot of area (several $\mathrm{mm}^{2}$ ) in the transceiver, even when monolithically integrated with the driver [13]. These traveling-wave structures typically require $50 \Omega$ terminations in the driver(s) and on the PIC, increasing the TX power consumption considerably. More compact single-modulator solutions, e.g. electro-absorption modulated lasers (EAEMLs)[6], and directly modulated lasers (DMLs)[7] often rely on power-hungry electrical digital-to-analog converters (DACs) to produce a (predistorted) PAM-4 signal which has to be linearly converted into the optical domain by the modulator. These transmitters typically use significant digital signal processing (DSP) at the TX or RX, or both. In [8], 56 GBd PAM-4 was transmitted with a single electroabsorption modulator (EAM) driven by an electrical DAC and RF-amplifier while using transmitter (TX) and receiver (RX) DSP, resulting in bit error ratios (BERs) below the HDFEC threshold $\left(3.8 \times 10^{-3}\right)$ up to $2 \mathrm{~km} \mathrm{SSMF.} \mathrm{In} \mathrm{[9],} \mathrm{a} \mathrm{single}$ microring modulator (MRR) and an integrated laser are combined with a low-power $(160 \mathrm{~mW})$ CMOS driver with on-chip compensation for the MRR characteristics. This resulted in 56 GBd PAM-4 with a very good eye quality, however without fiber transmission or BER. Out of [3-14], only [10], [11-12] and [14] utilize PAM-4 generation in the optical domain (optical DAC). In [10], up to 64 GBd PAM-4 is demonstrated with a segmented microring resonator (MRR), without driver. In [14], a low-power transmitter is demonstrated based on flip-chip integration of a CMOS driver on a segmented MZM, but it is limited to $28 \mathrm{GBd}$. In [12], we demonstrated 64 GBd PAM-4 transmission over $1 \mathrm{~km}$ SSMF in real-time, using a parallel configuration of NRZ-driven EAMs. However a combination of RF-probes, an analog TX FFE $(850 \mathrm{~mW})$ and RF-amplifiers (5W) was used to drive the EAMs, due to the lack of an EAM driver IC. In this paper, a photonic integrated circuit (PIC) from imec's iSiPP50G platform with parallel EAMs incorporated in a Mach-Zehnder interferometer (MZI) is wirebonded to a very low-power EAM driver IC [15], implemented in $55 \mathrm{~nm} \mathrm{SiGe}$ BiCMOS. The PIC contains a tunable optical power splitter and two lumped, unterminated EAMs, as was proposed in [11] but not yet implemented in [12]. The linearity requirements are removed from both driver and modulator, resulting in a low TX power consumption of only $160 \mathrm{~mW}$ (excluding laser), including the DC power dissipated in the heaters on the PIC (less than $15 \mathrm{~mW}$ ). The EAMs are very compact, each EAM occupying only 120x180 $\mu^{2}$ including metal routing and bondpads. BERs below or close to the KP4-FEC-threshold $\left(2.4 \times 10^{-4}\right)$ are shown for transmission of 53 GBd PAM-4 at $1565 \mathrm{~nm}$ across more than $1 \mathrm{~km}$ of SSMF and $2 \mathrm{~km}$ of non-zero dispersion-shifted fiber (NZ-DSF), while consuming only $1.5 \mathrm{pJ} / \mathrm{b}$ and occupying $0.275 \times 1.72$ $\mathrm{mm}^{2}$ on the PIC and $0.75 \times 1.2 \mathrm{~mm}^{2}$ on the EIC.

\section{Experiment setup}

The measurement setup is illustrated in Fig. 1. The operation principle of the transmitter, described in detail in [11] and [12], is shown in Fig. 2. Two PRBS $2^{9}-1$ pseudo-random bit sequences (PRBS) at $53 \mathrm{~Gb} / \mathrm{s}$, produced by a $92 \mathrm{GSa} / \mathrm{s}$ arbitrary waveform generator (AWG) and decorrelated by introducing a relative delay (of at least 20 symbol periods) in the AWG, are sent directly through a 6-inch multi-coax cable 

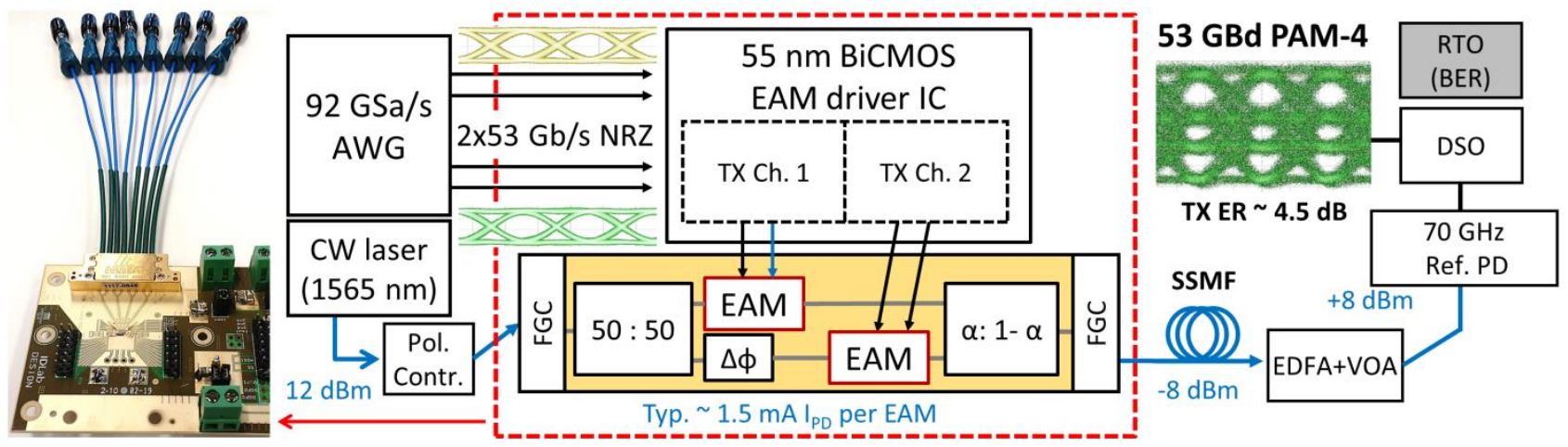

Fig. 1. Experiment setup.

assembly and RF-PCB transmission lines to the differential inputs of two driver channels in the TX electrical IC (EIC). The TX EIC non-linearly amplifies the input signals and drives the EAMs with up to $2 \mathrm{Vpp}$ differential swing. The wirebond length towards the EAMs is minimized in the assembly to improve bandwidth and reduce bondwireinduced peaking (see Fig. 2). The external laser, set to 12 $\mathrm{dBm}$ at $1565 \mathrm{~nm}$, is coupled into and out of the PIC through fiber-to-chip grating couplers (FGCs, $6 \mathrm{~dB}$ loss/FGC). Coupling loss can be improved to $2 \mathrm{~dB}$ per coupler with edge couplers [15]. A 50/50 power splitter is used at the input, ensuring that the EAMs receive equal optical power and behave as identical loads towards the driver IC. One branch is

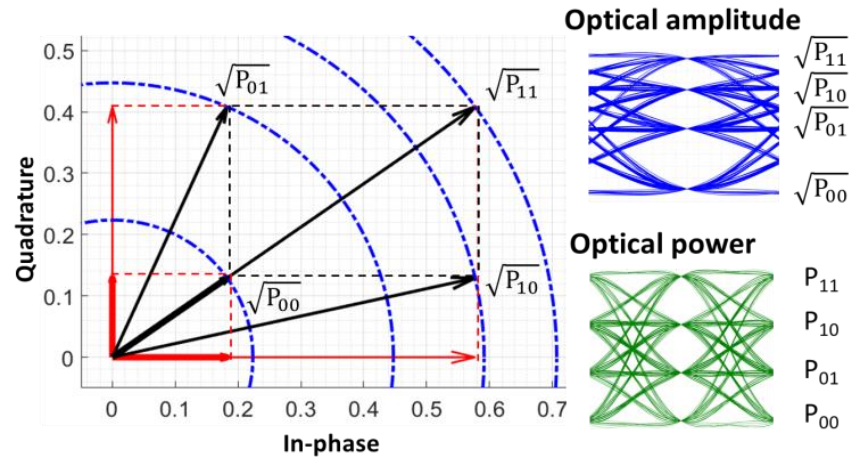

Fig. 2. PAM-4 transmitter principle, with $\Delta \phi=90^{\circ}$ and $\alpha=1 / 3$.

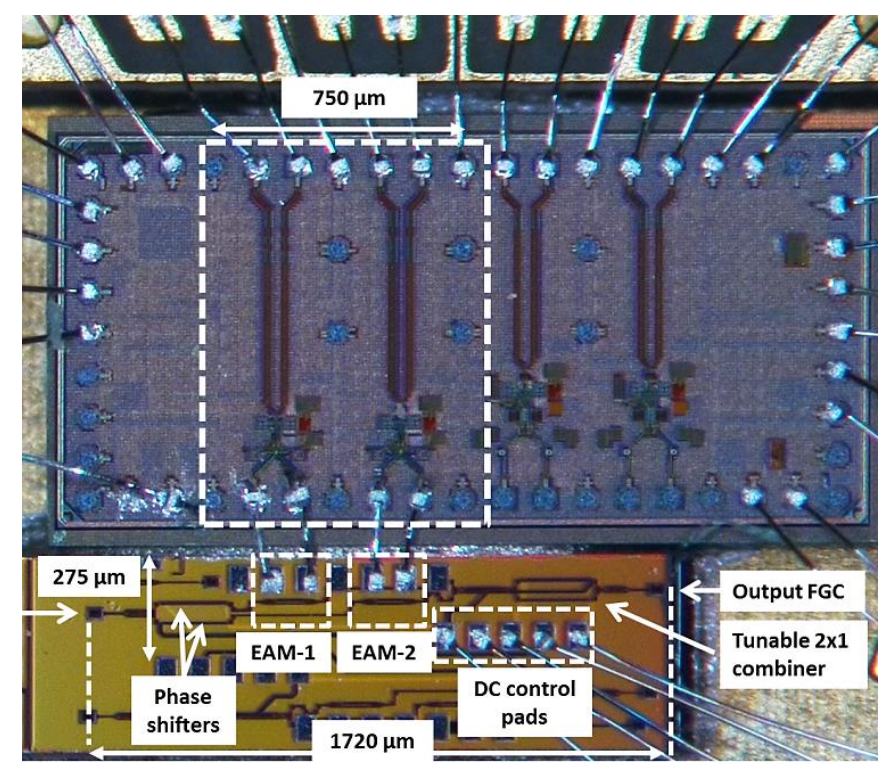

Fig. 3. Wirebonded PAM-4 TX. phase-shifted ( $\Delta \phi$ in Fig. 1-2) w.r.t. the other branch with a thermo-optic phase shifter. By default, the driver EIC biases both EAMs identically at approx. $-1 \mathrm{~V}$, resulting in approximately $1.5 \mathrm{~mA}$ DC photocurrent per EAM. The insertion loss per EAM was approximately $5 \mathrm{~dB}$. Next, the modulated light is recombined using a tunable power combiner (implemented as a balanced MZI). Nominally the MZI is set to 33/66-operation ( $\alpha=1 / 3$, Fig. $1-2)$, theoretically resulting in equidistant PAM-4 after power detection by the photodetector (PD) (Fig. 2) [11]. Thanks to the tunable optical recombination, up to $1.25 \mathrm{~dB}$ OMA improvement is expected w.r.t. an electrically weighted equivalent, as shown in [12]. After transmission over SSMF, an Erbium-doped fiber amplifier (EDFA) is used to overcome the insertion loss of the FGCs and as part of the reference receiver, which further consists of a variable optical attenuator (VOA) and a $70 \mathrm{GHz}$ PD. The PD output is captured by a digital sampling oscilloscope (DSO) for eye diagrams, or by a $63 \mathrm{GHz} 160$ $\mathrm{GSa} / \mathrm{s}$ real-time oscilloscope (RTO) for offline BER measurements. BER is measured directly after resampling, without using DSP.

\section{$3 \quad$ Results}

In Fig. 4, the TX eyes diagrams, measured with the reference $\mathrm{RX}$, are plotted vs. data rate. The TX outer extinction ratio

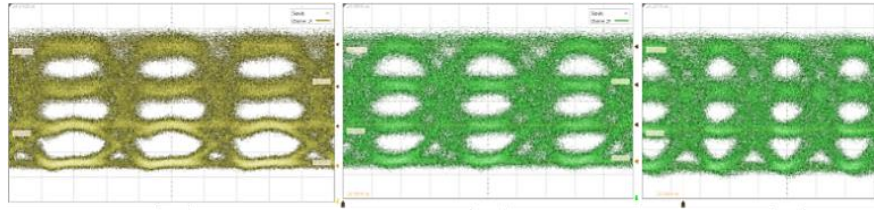

(a)

(b)

(c)

Fig. 4. TX output at (a) 28GBd (12ps/div, 15mV/div);

(b) $40 \mathrm{GBd}(8 \mathrm{ps} / \mathrm{div}, 15 \mathrm{mv} / \mathrm{div})$ and

(c) $53 \mathrm{GBd}(8 \mathrm{ps} / \mathrm{div}, 16 \mathrm{mV} / \mathrm{div})$.

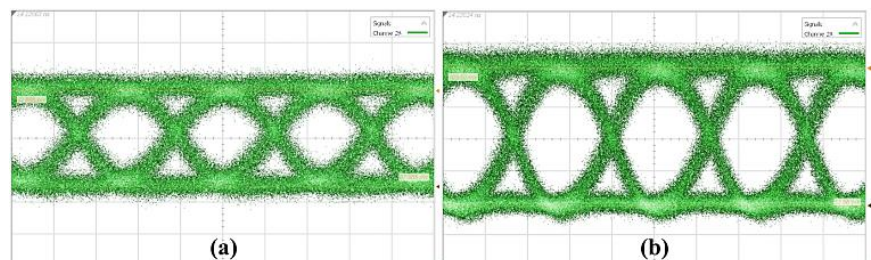

Fig. 5. TX NRZ outputs at $53 \mathrm{~Gb} / \mathrm{s}$. (a) LSB, $10 \mathrm{mV} / \mathrm{div}$, $8 \mathrm{ps} / \mathrm{div}$ (b) MSB, $12 \mathrm{mV} / \mathrm{div}, 8 \mathrm{ps} / \mathrm{div}$. 


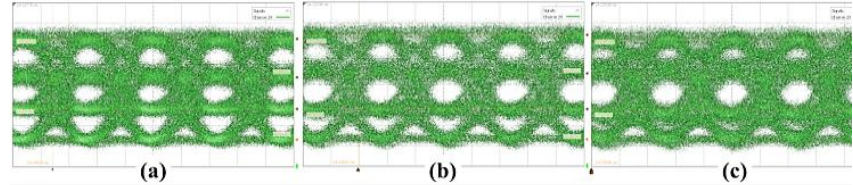

Fig .6. 53GBd TX output vs. SSMF fiber length: no predistortion ( $\alpha \approx 1 / 3$ ) (a) $0 \mathrm{~km}$, (b) $0.5 \mathrm{~km}$ (c) $1 \mathrm{~km}$

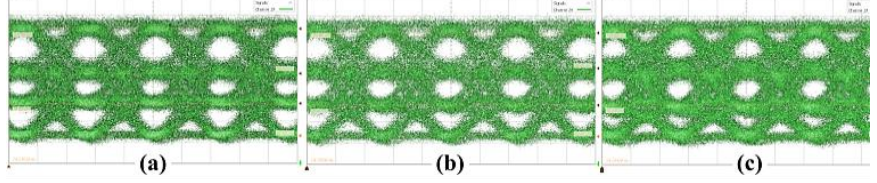

Fig .7. 53GBd TX output vs. SSMF fiber length: predistorted $(\alpha<1 / 3)(a) 0 \mathrm{~km}$, (b) $0.5 \mathrm{~km}$ (c) $1 \mathrm{~km}$

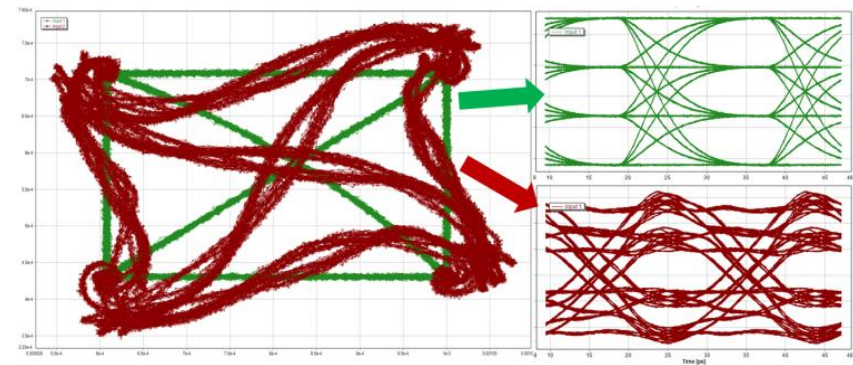

Fig. 8. Simulated TX output constellations and eye diagrams in back-to-back and with $1 \mathrm{~km}$ fiber.

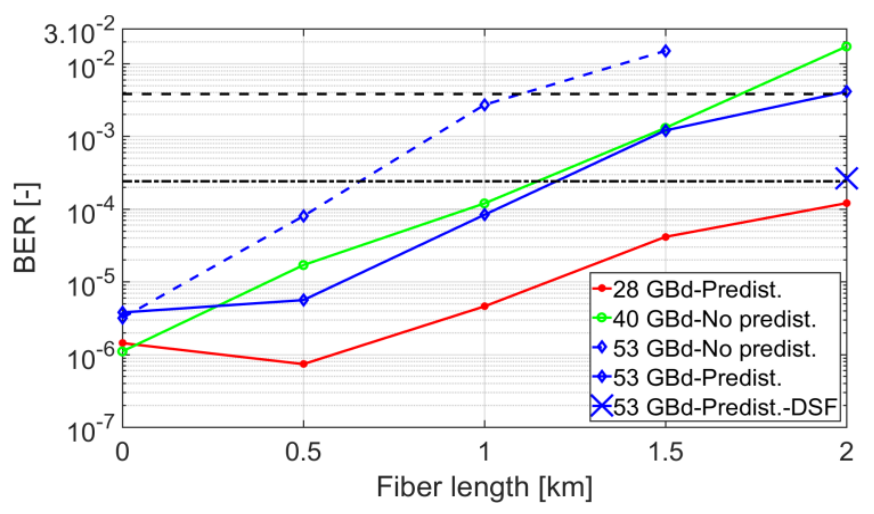

Fig.9. TX BER vs. fiber length

$\left(\mathrm{P}_{11} / \mathrm{P}_{00}\right.$, Fig. 2) is $5.3 \mathrm{~dB}$ at $28 \mathrm{GBd}$, and $4.5 \mathrm{~dB}$ at $40 \mathrm{GBd}$ and $53 \mathrm{GBd}$. For comparison, the $53 \mathrm{GBd}$ NRZ eyes, obtained by driving only MSB or LSB, are shown in Fig. 5. In Fig. 6-7, the 53GBd TX eyes diagrams, measured with the reference RX, are plotted vs. fiber length. Fig. 6. (a) shows the back-to-back TX output, optimized to obtain equidistant PAM-4 ( $\alpha \approx 1 / 3$ with $\alpha: 1-\alpha$ the power combination ratio of the tunable MZI). Chromatic dispersion results in, among other effects, symmetrical compression of the outer PAM-4 eyes which significantly increases the BER. VPI ${ }^{\circledR}$ simulation confirms this behaviour: with increasing fiber length, the outer edges of the constellation diagram are rotated w.r.t. its original center, which is located in the upper right quadrant of the complex plane (Fig. 2). This does not significantly affect $\mathrm{P}_{11}-\mathrm{P}_{00}$, but primarily reduces the eye opening in the outer eyes after detection by the PD. The tunable MZI on the PIC can be used to compensate this effect by changing only the DC heater voltage (and hence $\alpha$ in Fig. 1), which predistorts the TX PAM-4 output. This results in Fig. 7. As shown in Fig. 9, this improves the BER by approx. one order of magnitude and almost doubles the sub-KP4-FEC fiber reach to approx. $1.25 \mathrm{~km}$. Only a minimal BER penalty is observed in the back-to-back case: at 53GBd, a back-to-back BER of $3.77 \times 10^{-6}$ is obtained with predistortion vs. a BER of $3.17 \times 10^{-6}$ for the equidistant case. However without MZI predistortion, the BER increases above the KP4-threshold beyond $500 \mathrm{~m}$, whereas with predistortion, the BER at $1 \mathrm{~km}$ SSMF is still $8.3 \times 10^{-5}$. With $2 \mathrm{~km} \mathrm{SSMF,} \mathrm{a} \mathrm{BER} \mathrm{of} 4.1 \times 10^{-3}$ is measured, just above the HD-FEC threshold $\left(3.8 \times 10^{-3}\right)$. With $2 \mathrm{~km} \mathrm{NZ-DSF}$ (single point), the BER is $2.62 \times 10^{-4}$, just above KP4-FEC. At $40 \mathrm{GBd}$, the back-to-back TX BER (unpredistorted) decreases to $1.1 \times 10^{-6}$, but the BER increase vs. fiber length is similar to the unpredistorted $53 \mathrm{GBd}$ case. Except for the 40GBd back-to-back case $\left(1.1 \times 10^{-6}\right)$, the BER at $53 \mathrm{GBd}$ with predistortion is better than at $40 \mathrm{GBd}$ without predistortion. For $28 \mathrm{GBd}$ with predistortion, the back-to-back BER $\left(1.43 \times 10^{-6}\right)$ is slightly higher than the BER minimum of $7.35 \times 10^{-7}$ at $500 \mathrm{~m}$, due to the predistortion which results in a smaller center eye. For 28GBd, the BER is below KP4-FEC beyond $2 \mathrm{~km} \mathrm{SSMF}\left(1.2 \times 10^{-4}\right)$, and below HD-FEC up to 3.5 $\mathrm{km}$ SSMF $\left(1.9 \times 10^{-3}\right)$. Table 1 summarizes the state of the art on integrated PAM-4 transmitters. Compared to singlemodulator solutions, the TX generates PAM-4 optically from two NRZ-driven EAMs. Therefore no linear drivers are required and the non-linearity of the modulator does not need to be compensated (as in [17]), which can reduce the power consumption of the TX significantly. Compared to classical MZM-based transmitters [13], even with very compact MZMs $(\sim 750 \mu \mathrm{m}$ length in [14]), the TX is very low-power and compact, occupying only $0.275 \times 1.72 \mathrm{~mm}^{2}$ on the PIC.

\begin{tabular}{ccccc}
\hline Ref. & $\begin{array}{c}\text { Data rate } \\
{[\mathbf{G B d}]}\end{array}$ & $\begin{array}{c}\text { Power } \\
{[\mathbf{p J} / \mathbf{b}]}\end{array}$ & $\begin{array}{c}\text { TDECQ } \\
{[\mathbf{d B}]}\end{array}$ & $\begin{array}{c}\text { BER } \\
{[-]}\end{array}$ \\
\hline$[13]$ & 25 & 30 & - & - \\
\hline$[17]$ & 28 & 18 & - & $10^{-6}$ \\
\hline$[14]$ & $28 / 25$ & $1.59 * *$ & - & $-/ 2 \times 10^{-5 *}$ \\
\hline$[9]$ & 56 & $1.42^{* *}$ & 0.5 & - \\
\hline $\begin{array}{c}\text { This } \\
\text { work }\end{array}$ & 53 & $1.5^{* * *}$ & - & $3.17 \times 10^{-6}$ \\
\hline
\end{tabular}

Table 1. State-of-the art integrated PAM-4 transmitters *At 25 GBd. 28 GBd BER > KP4-FEC due to RX.

** Excluding heater(s) or temp. control. *** Including heaters

\section{Conclusion}

We have presented a $53 \mathrm{GBd}$ silicon integrated PAM-4 optical transmitter, capable of sub-KP4-FEC BERs over up to more than $1 \mathrm{~km}$ SSMF at $1565 \mathrm{~nm}$ and a BER just above KP4-FEC with $2 \mathrm{~km}$ NZ-DSF, without using any equalization or DSP. Compared to the state of the art, the transmitter is both very compact and low-power $(1.5 \mathrm{pJ} / \mathrm{b})$.

\section{$5 \quad$ Acknowledgements}

The work was supported by EU H2020 projects Streams, Teraboard and Picture. The work of J. Lambrecht and H. Ramon was supported by the FWO. 


\section{References}

[1] "IEEE P802.3bs", http://www.ieee802.org/3/bs, Accessed $05 / 03 / 2019$

[2] "100G Lambda Multi-Source Agreement", http://100glambda.com, Accessed 05/03/2019

[3] Chiuchiarelli, A., Ghandhi, R., Rossi, S. M. et al., "Single wavelength $100 \mathrm{G}$ real-time transmission for high-speed data center communications," Proc. Opt. Fiber Comm. Conf. (OFC), Los Angeles, CA, USA, 2017, WI4.2, pp. 1-3. [4] El-Fiky, El., Samani, A., Patel, D. et al., " 400 Gb/s Oband silicon photonic transmitter for intra-datacenter optical interconnects," Opt. Express, vol. 27, no. 7, pp. 10258 10268, April 2019.

[5] Lee, J., Shahramian, S., Kaneda, N. et al., "Demonstration of 112-Gbit/s Optical Transmission using 56GBaud PAM-4 Driver and Clock-and-Data Recovery ICs", Proc. European Conf. on Opt. Comm (ECOC), Valencia, Spain, 2015, DOI: 10.1109/ECOC.2015.7341667, pp. 1-3. [6] Nakai, Y., Nakanishi, A., Yamaguchi, Y. et al., "Uncooled Operation of 53-Gbaud PAM4 (106-Gb/s) EA/DFB Lasers with Extremely Low Drive Voltage with 0.9 Vpp,", Proc. European Conf. on Opt. Comm (ECOC), Rome, Italy, 2018, DOI: 10.1109/ECOC.2018.8535399, Mo4D.4, pp. 1-3.

[7] Sasada, N., Nakajima, T., Sekino, Y. et al., "Widetemperature-range $\left(25^{\circ} \mathrm{C}\right.$ to $\left.80^{\circ} \mathrm{C}\right) 53$-Gbaud PAM4 (106-

$\mathrm{Gb} / \mathrm{s})$ Operation of $1.3-\mu \mathrm{m}$ Directly Modulated DFB Lasers for 10-km Transmission", Proc. European Conf. on Opt. Comm (ECOC), Rome, Italy, 2018, PDP , pp. 1-3.

[8] El-Fiky, E., De Heyn, P., Osman, M. et al., "112 Gb/s PAM4 Transmission over $2 \mathrm{~km}$ SMF Using a C-band GeSi Electro-Absorption Modulator," Proc. Opt. Fiber Comm. Conf. (OFC), San Diego, CA, 2018, W2A.16, pp. 1-3. [9] Li, H., Balamurugan, G., Sakib, M., et al., “A 112 Gb/s PAM4 Transmitter with Silicon Photonics Microring Modulator and CMOS Driver", Proc. OFC 2019, Th4A.4, San Diego, USA, 2019

[10] Sun, J., Kumar, R., Sakib, M. et al., “A 128 Gb/s PAM4 Silicon Microring Modulator With Integrated Thermo-Optic Resonance Tuning”, in Journal of Lightwave Technology, vol. 37, no. 1, pp. 110-115, 1 Jan.1, 2019. doi:

10.1109/JLT.2018.2878327

[11] Verbist, J., Lambrecht, J., Verplaetse, M. et al., "DACless and DSP-free PAM-4 Transmitter at $112 \mathrm{~Gb} / \mathrm{s}$ with Two Parallel GeSi Electro-Absorption Modulators," 2017 European Conference on Optical Communication (ECOC), Gothenburg, 2017, pp. 1-3.doi:

10.1109/ECOC.2017.8346098
[12] Verbist, J., Lambrecht, J., Verplaetse, M et al., "RealTime and DSP-Free 128 Gb/s PAM-4 Link Using a Binary Driven Silicon Photonic Transmitter" in Journal of Lightwave Technology, vol. 37, no. 2, pp. 274-280, 15 Jan.15, 2019. doi: 10.1109/JLT.2018.2877461

[13] Rito, P., Lopez, I.G., Petousi, D. et al., "A

Monolithically Integrated Segmented Linear Driver and Modulator in EPIC 0.25- $\mu \mathrm{m}$ SiGe:C BiCMOS Platform," in IEEE Transactions on Microwave Theory and Techniques, vol. 64, no. 12, pp. 4561-4572, Dec. 2016.

[14] Tanaka, S., Takasi, S., Aoki, T. et al., "Ultralow-Power (1.59 mW/Gbps), 56-Gbps PAM4 Operation of Si Photonic Transmitter Integrating Segmented PIN Mach-Zehnder Modulator and 28-nm CMOS Driver", in Journal of Lightwave Technology, vol. 36, no. 5, pp. 1275-1280, March 1, 2018. doi: 10.1109/JLT.2018.2799965

[15] Ramon, H., Lambrecht, J., Verbist, J. et al., "70 Gb/s Low-Power DC-coupled NRZ Differential ElectroAbsorption Modulator Driver in 55 nm SiGe BiCMOS," in Journal of Lightwave Technology, vol. 37, no. 5, pp. 15041514, 1 March, 2019. doi: 10.1109/JLT.2019.2900192 [16] Snyder, B., Mangal, N., Lepage, G. et al., "Packaging and Assembly Challenges for 50G Silicon Photonics Interposers," Proc. Opt. Fiber Comm. Conf. (OFC), San Diego, CA, 2018, Tu2A.3, pp. 1-3.

[17] Kishi, T., Nagatani, M., Kanazawa, S. et al., "56-Gb/s Optical Transmission Performance of an InP HBT PAM4 Driver Compensating for Nonlinearity of Extinction Curve of EAM," in Journal of Lightwave Technology, vol. 35, no. 1, pp. 75-81, 1 Jan.1, 2017. doi: 10.1109/JLT.2016.2624778 